EPJ Web of Conferences 21, 08008 (2012)

DOI: $10.1051 /$ epjconf/20122108008

C Owned by the authors, published by EDP Sciences, 2012

\title{
Theoretical and experimental studies of the neutron rich fission product yields at intermediate energies.
}

\author{
V. A. Rubchenya ${ }^{1,2, a}$, D. Gorelov ${ }^{1}$, H. Penttilä ${ }^{1}$, and J. Äystö ${ }^{1}$ \\ ${ }^{1}$ Department of Physics, P.O. Box 35 (YFL), FI-40014 University of Jyväskylä, Finland \\ ${ }^{2}$ V.G. Khlopin Radium Institute, 194021, St. Petersburg, Russia
}

\begin{abstract}
A new method to measure the fission product independent yields employing the ion guide technique and a Penning trap as a precision mass filter, which allows an unambiguous identification of the nuclides is presented. The method was used to determine the independent yields in the proton-induced fission of ${ }^{232} \mathrm{Th}$ and ${ }^{238} \mathrm{U}$ at $25 \mathrm{MeV}$. The data were analyzed with the consistent model for description of the fission product formation cross section at the projectile energies up to $100 \mathrm{MeV}$. Pre-compound nucleon emission is described with the two-component exciton model using Monte Carlo method. Decay of excited compound nuclei is treated within time-dependent statistical model with inclusion of the nuclear friction effect. The charge distribution of the primary fragment isobaric chain was considered as a result of frozen quantal fluctuations of the isovector nuclear density. The theoretical predictions of the independent fission product cross sections are used for normalization of the measured fission product isotopic distributions.
\end{abstract}

\section{Introduction}

Nuclear fission is a promising source for discovering, producing, and investigating exotic nuclei with high neutron excess [1]. Precise measurements of the fission product distributions deliver valuable information on the formation of compound fissioning nucleus and on the dynamics of the large-scale collective motion of nuclear matter. Comparative investigations of neutron-rich fission product yields in fission of heavy nuclei induced by deuterons, protons, neutrons and photons in wide range of energies are important for the development of radioactive beam facilities such as EURISOL [2]. Reliable predictions of fission product yields are also important for the technical application of nuclear reactions at intermediate energy for the energy production and transmutation of nuclear waste in hybrid reactors [3] and for developing advanced new generation nuclear GEN-IV reactors [4].

ae-mail: rubchen@phys.jyu.fi

This is an Open Access article distributed under the terms of the Creative Commons Attribution-Noncommercial License 3.0, which permits unrestricted use, distribution, and reproduction in any noncommercial medium, provided the original work is properly cited. 


\section{EPJ Web of Conferences}

New method to measure independent fission product yields was developed recently at the Accelerator Laboratory of the University of Jyväskylä [5]. This method combines the capability of the ion guide (IGISOL) technique and high mass resolving power of Penning trap (JYFLTRAP). It is thus applicable to all elements produced in fission.

To analyze experimental results, a new version of the theoretical model for calculation of the light particle induced fission characteristics has been developed. This model is a combination of new version of the two-component exciton model [6] and a time-dependent statistical model for fusion-fission process with inclusion of dynamical effects [7] for accurate calculations of nucleon composition and excitation energy of the fissioning nucleus at the scission point. The model for calculation of fission product yields proposed earlier [8] was upgraded by including a dynamical approach for isobaric width and calculating the parameters of charge polarization at scission point.

In this report a description of the novel method for measuring independent fission product yields is shortly described. A description of model for calculations of fission product cross sections in the fission of heavy nuclei induced by light particles with energies up to 100 $\mathrm{MeV}$ is presented. The comparison between the theoretical predictions and experimental data obtained with JYFLTRAP for the $25 \mathrm{MeV}$ proton-induced fission of ${ }^{232} \mathrm{Th}$ and ${ }^{238} \mathrm{U}$ will be done.

\section{Independent yield measurements with a Penning trap}

The main feature of the method is that a Penning trap is used as a precision mass filter, which allows identifying each nuclide on its mass. To separate nuclides of the fission product isobaric chain with the mass number $A$, it is needed a mass resolving power $m / \Delta m>5000$. A mass resolving power higher than 5000 is not easy to be achieved for any magnetic mass separator. Therefore, isotopes with the same $A$ are usually resolved via detection of their radioactive decays. It leads to low sensitivity, long measurement and a large uncertainty due to incomplete decay schemes and accumulation of certain isotopes via the $\beta$-decay of their parent nuclei.

A schematic view of the experimental setup is presented in figure 1. In general, the facility consists from two big parts: IGISOL (Ion Guide Isotope Separator On Line) mass separator and the double Penning trap - JYFLTRAP. The IGISOL provides selection of ions with certain mass number $A$, whereas JYFLTRAP is responsible for nuclide identification.

Ions produced in a nuclear reaction are stopped by the noble buffer gas. Due to high ionization potential of the buffer gas, considerable part of ions acquires charge state +1 after slowing down. Ions are extracted from the ion guide by gas flow and electric field. The extracted ions are accelerated up to $30 \mathrm{q} \mathrm{keV}$ and the formed continuous beam is guided through a dipole magnet separator with the resolving power around $\mathrm{m} / \Delta \mathrm{m} \approx 300$. As result the isobaric chain with certain mass number $A(\mathrm{q}=+1)$ is selected. After selection ions are injected into the radio frequency quadrupole cooler/buncher (RFQ cooler/buncher) where they are stopped, cooled, and stored as a bunch. Short bunch is released to a double Penning trap.

JYFLTRAP consists of two equal cylindrical Penning traps placed in superconducting magnet with very uniform magnetic field around $7 \mathrm{~T}$. However, for the yield measurements only so called purification trap is used together with a buffer gas-cooling technique [20]. When ions are inside the trap, the following excitation scheme is applied. Full cycle starts from dipole excitation. Due to this all ions independently from their masses are moved to a large radius. After that applying quadrupole excitation with frequency close to a cyclotron 
frequency, only ions with certain mass are focused in the center of the trap. The relation between ion mass and cyclotron frequency is given by equation:

$$
f_{c}=\frac{1}{2 \pi} \frac{q}{m} B
$$

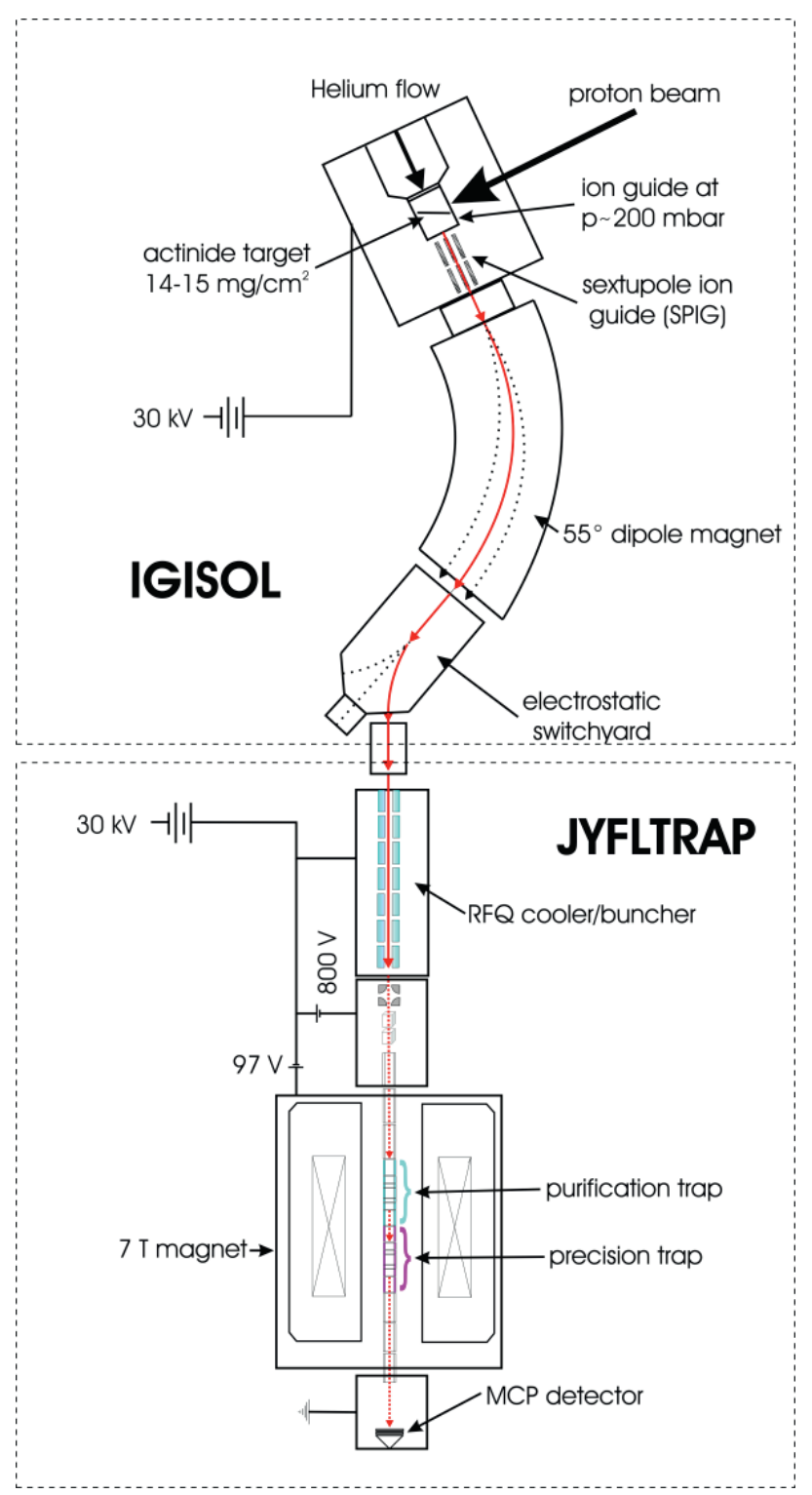

Fig. 1. A schema of the experimental apparatus (not to scale): IGISOL (upper part of schema) provides extraction and selection of isobaric chain with mass number $A$ and JYFLTRAP (lower part) separates nuclides with certain $Z$, which are registered by MCP detector. 


\section{EPJ Web of Conferences}

Focused ions are released from the trap through $2 \mathrm{~mm}$ diaphragm and registered by a micro channel plate (MCP) detector. To reduce the noise background, the time of flight from the trap to MCP detector is measured for every detected ion in the bunch. Thereby the mass spectrum is generated by counting how many ions were detected for each quadrupole excitation frequency.

For one measurement cycle with JYFLTRAP it takes some hundreds milliseconds. That's why the method is suitable for registration of nuclides with very small half-life up to around 100 milliseconds.

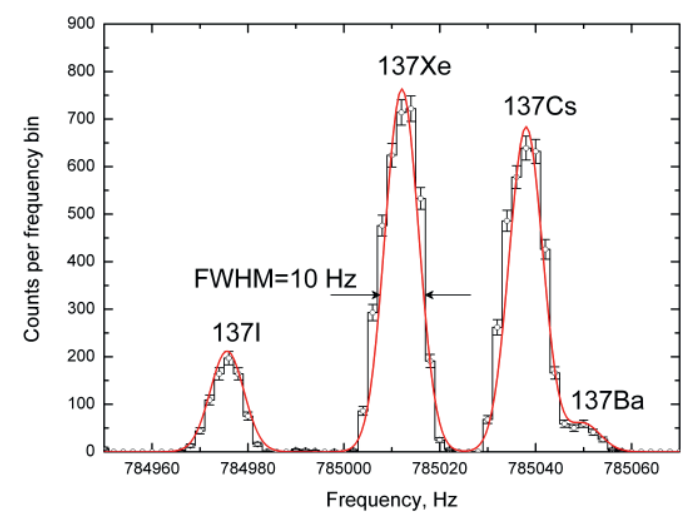

Fig. 2. Number of ions counted by MCP detector as function of quadrupole frequency for isobaric chain $\mathrm{A}=137$. The histogram with circles is an experimental spectrum. Smooth line presents a fit with four Gaussians.

Typical MCP count numbers (histogram with circles and error bars) during the quadrupole frequency scanning for isobaric chain $A=137$ is presented in figure 2. Approximation with four Gaussians is shown by a line. Count numbers at the Gaussian centroids are proportional to the nuclide yields, and the Gaussian dispersion characterizes the mass resolution $\Delta m$. Estimated mass resolving power of purification Penning trap for mass number 137 is $m / \Delta m=7.8 \cdot 10^{4}$.

The counting rate of each isotope is determined from the peak intensity of mass spectrum. However, it is quite difficult to get absolute cross-section from this measurements. The main reason for that is the unknown absolute efficiency of the setup. The ion guide technique is chemically unselective, but the absolute efficiency for different elements is not the same. Nevertheless in the context of this method, it is possible to obtain relative yield distribution for isotopes of the same element. That is why to determine the independent yield, the counting rate of each isotope has to be measured together with the reference one. Finally, obtained relative distribution might be normalized on the known cross-section for any isotope.

Detail information about this method and accurate description of data analysis is given in the work [5].

\section{Theoretical model}

The detailed theoretical calculation of the fission product formation cross sections consists of three reaction stages description: (i) modeling the reaction mechanism to calculate mass, 


\section{$\mathrm{CNR} * 11$}

charge, and excitation energy distributions of compound nuclei; (ii) modeling the fission process itself and the primary fission fragment formation and (iii) modeling the deexcitation process of the heated primary fragments.

\subsection{Formation of the compound nuclei ansemble at the scission point}

The pre-equilibrium particle emission process is described in the frame of the twocomponent exciton model [6]. It is supposed that duration of the pre-equilibrium process is two orders of magnitude shorter than the average statistical decay time of the initial composite-nucleus.

After ending the pre-equilibrium stage for every partial wave in the entrance channel, a consideration of the decay of compound nuclei states starts. The pre-scission neutrons and other light particles are emitted near equilibrium deformation of the compound nucleus and at descent from saddle to scission point. The fission width and the descend time is strongly influenced by nuclear friction.

The Monte-Carlo simulation method was used to calculate a pre-scission neutron multiplicity and spectra using the compound nuclei parameters formed after the preequilibrium neutron and proton emission. The time-dependent statistical approach was applied for description of particle emission during the fission process up to scission point to take into consideration fission the delay time and finite descent time from saddle to scission [7]. After pre-scission particle emission, the compound nucleus arrives at the scission point with some distributions of excitation energy $E_{C N}$, mass $A_{C N}$, charge $Z_{C N}$, and spin $J_{C N}$.

For each member of this ansemble the primary fission fragment mass, charge and excitation energy distributions are calculated. The independent fission product formation cross sections are calculated by summing over all compound nuclei and their excitation energy at the scission point

$$
\sigma_{\text {ind }}(A, Z)=\sum_{A_{C N}, Z_{C N}} \int d \sigma_{C N}^{S C}\left(A_{C N}, Z_{C N}, E_{C N}\right) / d E_{C N} \cdot Y_{\text {ind }}\left(A, Z, A_{C N}, Z_{C N}, E_{C N}\right) d E_{C N},
$$

where $\sigma_{C N}^{S C}$ is a formation cross section of given compound nucleus at the scission point. The fission product independent yields are formed in the process of neutron evaporation from excited primary fragments and calculated according to equation

$$
Y_{\text {ind }}(A, Z)=\sum_{n} Y_{\text {pre }}(A+n) P_{Z}(A+n) P_{n}(A+n, Z) .
$$

Here $Y_{\text {pre }}$ is the primary isobaric chain yield, $P_{Z}$ is the charge distribution of the primary isobaric chain, and $P_{n}(A+n, Z)$ is the n-neutron emission probability from primary fission fragment $(Z, A+n)$.

\subsection{Charge distribution of the primary isobaric chains}

The charge distribution of the primary isobaric chain is presented in the factorized form

$$
P_{Z}(A)=\widetilde{P_{Z}}(A) F_{Z}^{o e}(A),
$$

where $\widetilde{P_{Z}}(A)$ is a smoothed distribution and $F_{Z}^{o e}$ describes odd-even staggering in the charge distribution. The smoothed distribution is usually approximated by a Gaussian function

$$
\widetilde{P_{Z}}(A)=\frac{1}{\sigma_{Z}(A) \sqrt{2 \pi}} \exp \left[-\frac{(Z-\bar{Z}(A))^{2}}{2 \sigma_{Z}^{2}(A)}\right] .
$$

The averaged charge deviates from the unchanged charge density distribution value 


$$
\bar{Z}(A)=A \frac{Z_{C N}}{A_{C N}}+\delta \bar{Z}(A)
$$

Charge polarization $\delta \bar{Z}$ is determined by the global liquid drop properties of the potential energy surface near the scission point and influenced by nuclear shell and pairing effects. To calculate the charge dispersion, $\sigma_{Z}(A)$ we shall consider the isobaric charge width as a result of a charge frozen quantal fluctuation at scission point. [9]. A fissioning nucleus at the scission point is described by two slightly overlapping fragments. The radius of the aperture $r_{\text {neck }}$ (neck radius) through which the two fragments may exchange nucleons is about $2 \mathrm{fm}$. It is assumed that the isovector nuclear density degree of freedom is much faster than the deformation and mass ones. Therefore it is possible to fix fragment masses and study the variation of the fragment charge $Z$ alone.

For small oscillations, the charge collective motion is harmonic in the smoothed potential

$$
\tilde{V}(Z)=V(\bar{Z})+\frac{1}{2} C_{Z Z}(Z-\bar{Z})^{2} .
$$

The stiffness parameter $C_{Z Z}$ and average charge $Z$ for given mass fragments division are calculated in the framework of the scission point fission model. The scission point configuration is approximated by two nascent deformed fragments. Potential energy of the scission configuration consists of the interaction energy between fragments and their deformation energies. Deformation energy has been calculated using the Strutinsky macroscopic-microscopic method [10]. Nuclear shape was described in the lemniscates coordinate system where the Cassini ovals are used as basic figures [11]. The single particle spectra have been calculated in the axially deformed Woods-Saxon potential using universal nuclear potential parameters proposed in Ref. [12].

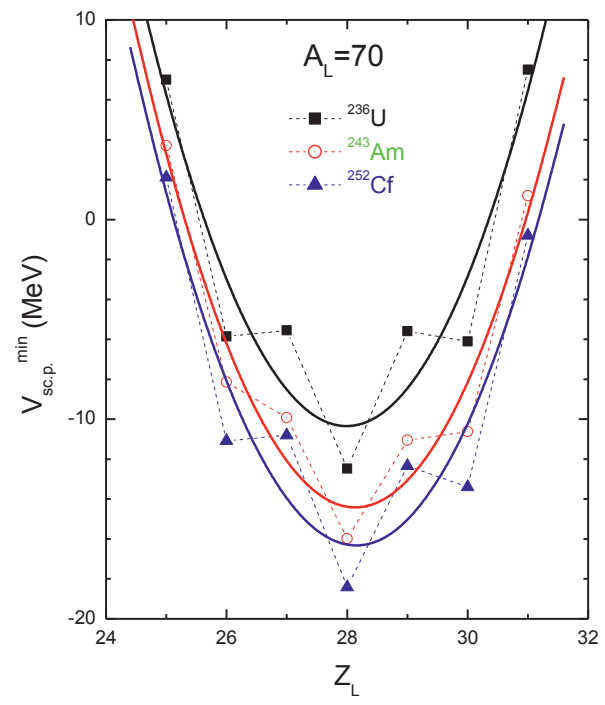

Fig. 3. Calculated charge dependences of potential energy for the isobaric chain with $A=70$ for compound nuclei ${ }^{236} \mathrm{U},{ }^{243} \mathrm{Am}$, and ${ }^{252} \mathrm{Cf}$. Parabolic approximation of calculated values are shown by curves. 


\section{$\mathrm{CNR} * 11$}

For a given compound nucleus after minimization of the potential relative to the deformation parameters of both fragments, the two-dimensional function of $V_{\min }(A, Z)$ was computed. The potential energy at fixed fragment mass was approximated by the parabolic dependence and parameters $\bar{Z}$ and $C_{Z Z}$ have been determined. As example, the calculated potential energy as a function of fragment charge of isobaric chain $A=70$ for ${ }^{236} \mathrm{U},{ }^{243} \mathrm{Am}$, and ${ }^{252} \mathrm{Cf}$ is shown in figure 3 where the points present computed values and curves are approximating functions. One can see from figure 3 that the nuclear shell $Z=28$ stabilizes the potential energy minimum for the wide range of compound nuclei.

The odd-even staggering and nuclear shell effect manifest in the dependence of $\bar{Z}(A)$ and $C_{Z Z}(A)$ relative to the values predicted by the liquid drop model as one can see from figure 4 where the calculated values (open squares) for spontaneous fission of ${ }^{239} \mathrm{~Np}$ are shown. With increasing excitation energy the paring and shell effects disappeared and the dependences are approaching the liquid drop limit (circles).

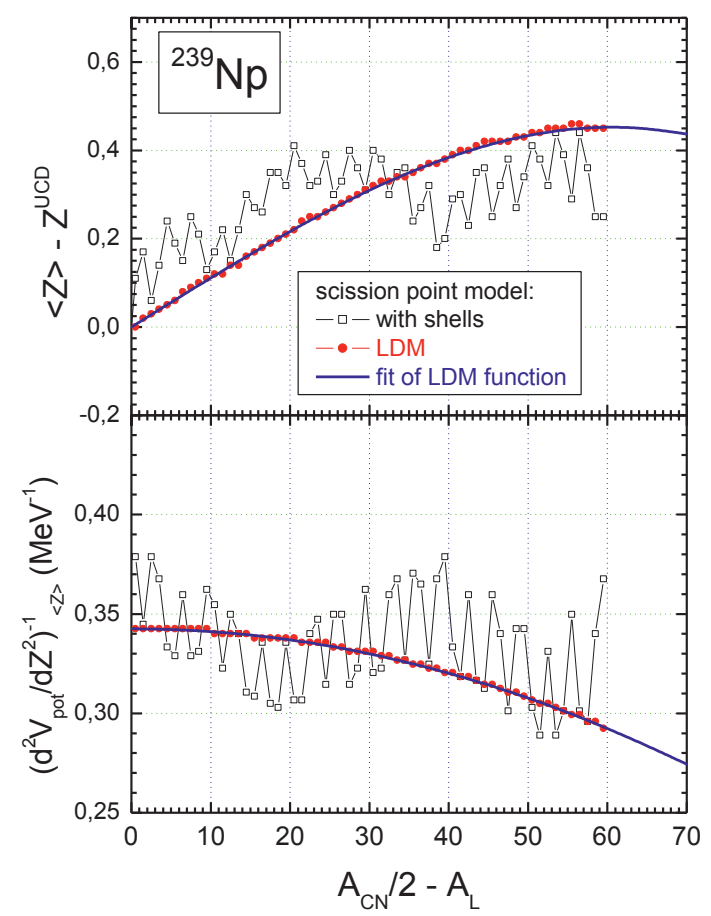

Fig. 4. Calculated values of $\delta \bar{Z}(A)$ and $C_{Z Z}^{-1}(A)$ for ${ }^{239} \mathrm{~Np}$ nucleus with inclusion of shell and pairing effects (open squares) and in the liquid drop model (circles). Fitting the liquid drop values are presented by the curves.

The standard deviation of the smoothed charge distribution in equation (4) is defined by the formula

$$
\sigma_{Z}(A)=\frac{1}{2} \frac{\hbar}{\sqrt{M_{Z Z} C_{Z Z}}}
$$


To calculate the inertia parameter the formula derived in Ref. [14] was used:

$$
M_{Z Z}=\frac{16}{9} r_{0}^{3} m \frac{A_{C N}}{Z_{C N} N_{C N}} \frac{l_{n e c k}+2 r_{n e c k}}{r_{n e c k}^{2}}
$$

Here $r_{0}$ is a nuclear radius parameter, $m$ is a nucleon mass, $l_{\text {neck }}$ is a neck length $\left(l_{\text {neck }}=\right.$ $2-4 \mathrm{fm})$, and $r_{\text {neck }}$ is a neck radius $\left(r_{\text {neck }}=2 \mathrm{fm}\right)$.

The odd-even correction function $F_{Z}^{o e}$ in the equation (4) was approximated by function

$$
F_{Z}^{o e} \propto \exp \left(\left(\Pi_{Z}^{H}+\Pi_{Z}^{L}\right) \delta_{Z}\left(A_{C N}, Z_{C N}, E_{C N}\right)\right.
$$

where the proton number parities in light $\Pi_{Z}^{L}$ and heavy $\Pi_{Z}^{H}$ fragments are equal to 1 (even $Z$ ) or -1 (odd $Z$ ). The proton odd-even differences parameter $\delta_{Z}\left(A_{C N}, Z_{C N}, E_{C N}\right)$ is parameterized in accordance with experimental data [13].

\subsection{Primary fragment mass distribution}

The pre-neutron emission isobaric chain yield is also presented in the factorized form

$$
Y_{\text {pre }}(A)=\tilde{Y}_{\text {pre }}(A) F_{A}^{o e} .
$$

Parameterization of smoothed mass distribution is based on the multimodal nature of nuclear fission [15], depicting the influence of nuclear-shell structure on the potential-energy surface (PES) of fissioning nucleus. The fission process is most probably guided by the valleys and bifurcation points of the PES from equilibrium shape to the scission point. For heavy actinides (from Th to $\mathrm{Cf}$ ), the so-called standard fission modes (symmetric, spherical ${ }^{132} \mathrm{Sn}$, and deformed $\mathrm{N}=86-90$ shells) have been used. To proceed into very asymmetric fragment mass region, two additional superasymmetric fission modes were added. The five fission modes: symmetric $(S Y)$, standard-I $(S I)$, standard-II $(S I I)$, superasymmetric-I $(S A I)$ and superasymmetric-II $(S A I I)$ were taken into consideration to approximate the smoothed primary mass distribution [16]:

$$
\begin{aligned}
\tilde{Y}_{p r e}(A)= & \sum_{m=1}^{5} C_{m}\left(A_{C N}, Z_{C N}, E_{C N}\right) Y_{m}(A)=C_{S Y} Y_{S Y}(A)+C_{S I} Y_{S I}(A)+C_{S I I} Y_{S I I}(A)+ \\
& C_{S A I} Y_{S A I}(A)+C_{S A I I} Y_{S A I I}(A) .
\end{aligned}
$$

Symmetric component $Y_{S Y}$ corresponds to a global liquid drop valley on PES in the multidimensional deformation parameter space. Nuclear shells modulate potential surface creating additional valleys which disappear at high compound nucleus excitation energy. The component $Y_{S I}$ is connected with $Z=50$ and $N=82$ nuclear shells in heavy fragments ( ${ }^{132} \mathrm{Sn}$-mode), and $Y_{S I I}$ component is influenced by "deformed" nuclear shell at $N=86-$ 90. The superasymmetric-I mode $Y_{S A I}$ and superasymmetric-II mode $Y_{S A I I}$ are supposed to be connected with neutron shell $N=50$, and proton shell $Z=28$ ( ${ }^{78} \mathrm{Ni}$-mode) in light fragments. In the first approximation, the mode components have a Gaussian form and 15 parameters are needed in the formula (12). The dependences of these parameters on the compound nuclei excitation energy has been parameterized using the experimental data from the proton induced fission of ${ }^{232} \mathrm{Th},{ }^{238} \mathrm{U}$, and ${ }^{242} \mathrm{Pu}$ measured with HENDES setup in Jyväskylä [17 - 19].

As example the prediction of the excitation energy dependences of the fission mode weights $C_{m}$ for ${ }^{239} \mathrm{U}$ compound nucleus are shown in figure 5 . In the case of the fast particle 
induced fission, the fragment mass yields are formed by contribution from different compound nuclei at scission point which have a slightly different energy dependences of mode weights $C_{m}$.

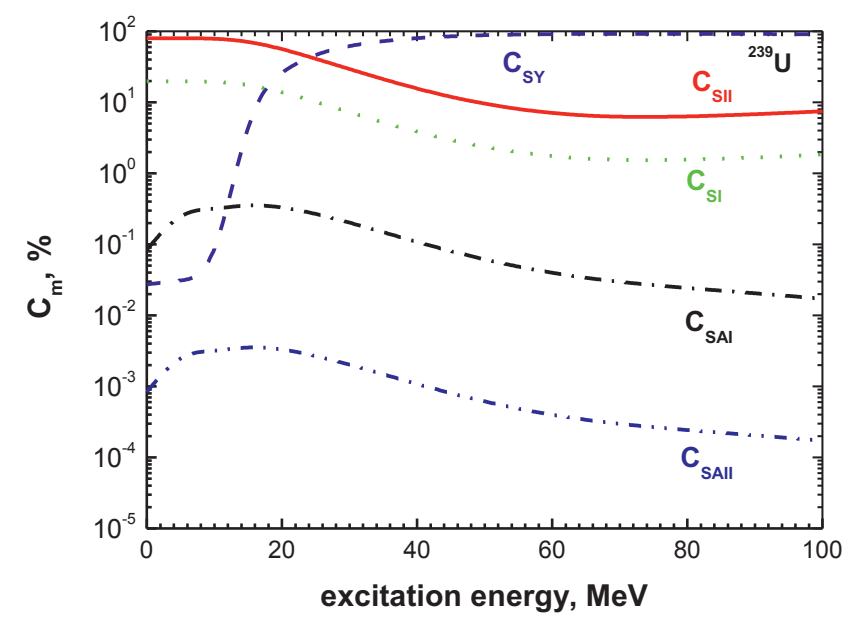

Fig. 5. The dependences of the fission mode weights on the excitation energy of ${ }^{239} \mathrm{U}$ compound nucleus.

Odd-even correction for primary fragment mass distribution is defined as

$$
F_{Z}^{o e} \propto \exp \left(\left(\Pi_{Z}^{H}+\Pi_{Z}^{L}\right) \delta_{Z}\left(A_{C N}, Z_{C N}, E_{C N}\right)+\left(\Pi_{N}^{H}+\Pi_{N}^{L}\right) \delta_{N}\left(A_{C N}, Z_{C N}, E_{C N}\right),\right.
$$

where the neutron and proton parities for both fragments are included. The neutron odd-even parameter $\delta_{N}$ is supposed to be half of the proton one.

\subsection{Post-scission neutron emission}

The calculation of the neutron multiplicity distribution for given primary fission fragments $P_{n}(A, Z)$ in the formula (2) is a difficult task because information about statistical properties and the excitation energy of fragments is still scarce. Here the simplified method was used, where the prompt neutron multiplicity distribution is approximated by the normal one

$$
P_{n}(A, Z)=\frac{1}{\sigma_{n}(A, Z) \sqrt{2 \pi}} \exp \left[-\frac{(n-\bar{n}(A, Z))^{2}}{2 \sigma_{n}^{2}(A, Z)}\right] .
$$

Standard deviation is approximated by linear function of averaged multiplicity and depends on excitation energy at scission point

$$
\sigma_{n}(A, Z)=0.75+0.21 \cdot \bar{n}(A, Z)
$$

Mean fragment excitation energies were calculated in the framework of the fission scission point model as described in Ref. 6. The averaged prompt neutron multiplicities of isobaric chains $\bar{n}(A)$ at the most probable charges have been calculated within standard statistical 
model taking into account dispersion of fragment excitation energy. The neutron multiplicities for different charge numbers of the isobaric chain were calculated using simple relations

$$
\begin{aligned}
\bar{n}(A, Z) & =\bar{n}(A) \frac{Q_{f}-c Z\left(Z_{C N}-Z\right)}{\overline{Q_{f}}-c\left(Z_{C N} \bar{Z}-\bar{Z}^{2}-\sigma_{Z}^{2}\right)}, \\
c & =\frac{1.44}{1.25\left(A^{1 / 3}+\left(A_{C N}-A\right)^{1 / 3}+2.5\right)},
\end{aligned}
$$

where $\overline{Q_{f}}$ is a mean value of the fission energy release for the isobaric chain.

Computer code FIPRODY (FIssion PRODuct Yield) was developed to calculate the characteristics of the light particles induced fission and photo-fission. Codes provide calculations of the following characteristics related to the prediction of the fission product yields:

- preequilibrium emission mean multiplicity of neutron and protons;

- prompt fission neutron spectra;

- pre- and post-scission multiplicities of light particles,

- pre-neutron emission fission fragment mass distributions;

- fission product isobaric chain yields;

- independent fission product yields.

\section{Experimental measurements}

Independent fission yield measurements for proton-induced fission of ${ }^{232} \mathrm{Th}$ and ${ }^{238} \mathrm{U}$ were carried out in the Accelerator Laboratory of the University of Jyväskylä, Finland. The method mentioned above was utilised.

Experimental procedures in both cases were very similar. However, there were some minor differences. First of all, in the experiment with ${ }^{238} \mathrm{U}$ target only one nuclide from isotopic chain was used as reference, whereas in the experiment with ${ }^{232} \mathrm{Th}$ some reference points were taken. Secondly, in the experiment with ${ }^{232} \mathrm{Th}$ an automatic control was used for 55 degree dipole magnet (see figure 1.), against the manual control in the run with ${ }^{238} \mathrm{U}$. Automatic control allowed us to reduce the dipole magnet instability and to remove the yield fluctuation with time.

Data analysis for ${ }^{238} \mathrm{U}$ fission experiment has almost done, but for ${ }^{232} \mathrm{Th}$ it's still in progress. That's why all experimental points presented in the report are preliminary.

\subsection{Independent fission yields from proton-induced fission of ${ }^{238} \mathrm{U}$ at $25 \mathrm{MeV}$}

Fission was induced via bombardment of a $15 \mathrm{mg} / \mathrm{cm}^{2}$ natural uranium target, placed in a standard fission ion guide (see details in [5]). The proton beam energy was $25 \mathrm{MeV}$ with intensity on the target about some $\mu \mathrm{A}$.

Experimentally determined and calculated independent isotopic yield distributions for bromine and caesium isotopic chains are shown in the figures 6 and 7. As mentioned in section 2 , in the frame of this method it is difficult to measure absolute cross sections. That's why the relative distribution was measured. After that the experimental points were fitted with Gaussian and the Gaussian's area was normalized to the calculated total yield. Experimental error includes all uncertainties described in [5]. The main sources of uncertainty are the intensity of the mass peak (statistical uncertainty), decay correction, irresolvable isomeric states and history correction. 


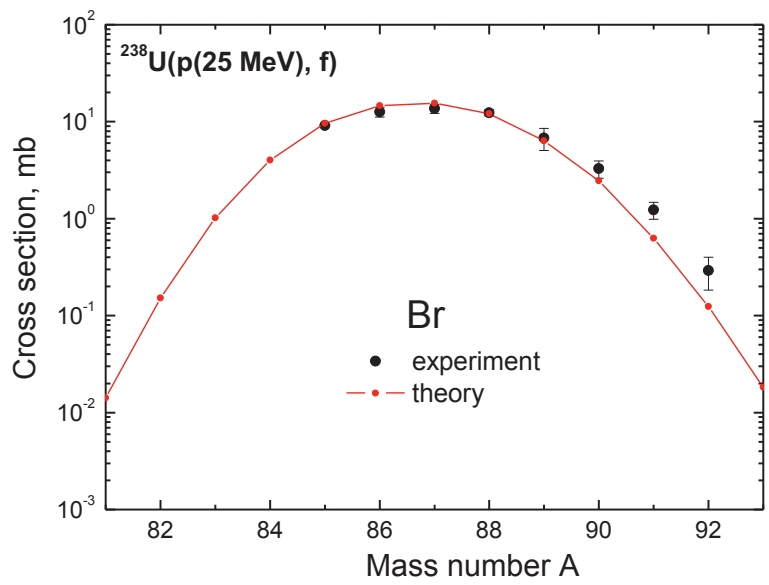

Fig. 6. Measured independent yields (dots with errors) of the bromine isotopes in the proton-induced fission of ${ }^{238} \mathrm{U}$ at $25 \mathrm{MeV}$. Theoretical prediction is shown by solid line. Experimental data were normalized to the calculated total yield of bromine (details see below in text).

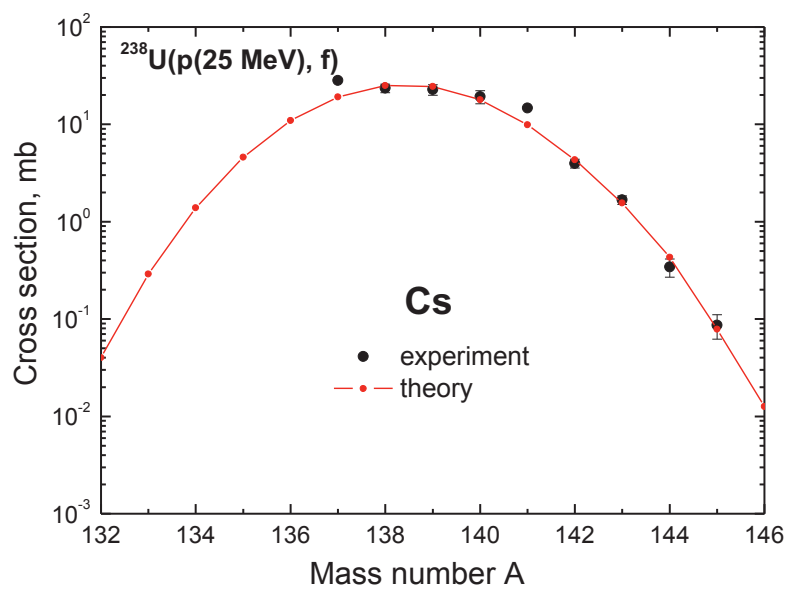

Fig. 7. The same as in figure 6 for the caesium isotopes.

\subsection{Independent fission yields from proton-induced fission of ${ }^{232} \mathrm{Th}$ at $25 \mathrm{MeV}$}

In this experiment ${ }^{232} \mathrm{Th}$ target (thickness $14 \mathrm{mg} / \mathrm{cm}^{2}$ ) was bombarded by the $25 \mathrm{MeV}$ proton beam. The same ion guide was used as in the experiment with the uranium target. Adding the automatic control for the dipole magnet significantly improved a quality of experimental data. In this modification it was possible to make so called "time distributed 


\section{EPJ Web of Conferences}

measurements", when the reference nuclide and measuring one changed each other more often. As result one facility provided almost parallel measurements for two isotopes.

Preliminary experimental data and theoretical calculations for bromine and caesium isotopes for ${ }^{232} \mathrm{Th}$ target are presented in the figures 8 and 9. Normalization for experimental points was done in the same manner as for ${ }^{238} \mathrm{U}$ fission.

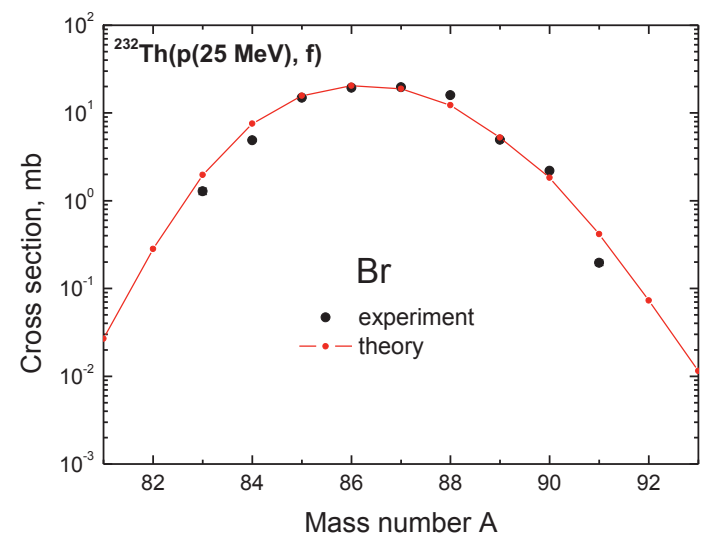

Fig. 8. As in figure 6 for the bromine isotopes in the proton-induced fission of ${ }^{232} \mathrm{Th}$ at $25 \mathrm{MeV}$.

In this case experimental uncertainty includes only statistical uncertainty, and decay correction. A problem with the yield fluctuation due to the dipole magnet instability was solved. That's why the history correction isn't required for the present data. A contribution from irresolvable isomeric states will be done later. However, this will not change bromine and caesium distributions significantly, because half-life of isomeric states of bromine and caesium isotopes are much longer or shorter, than the typical measurement time (tenths of second - some seconds).

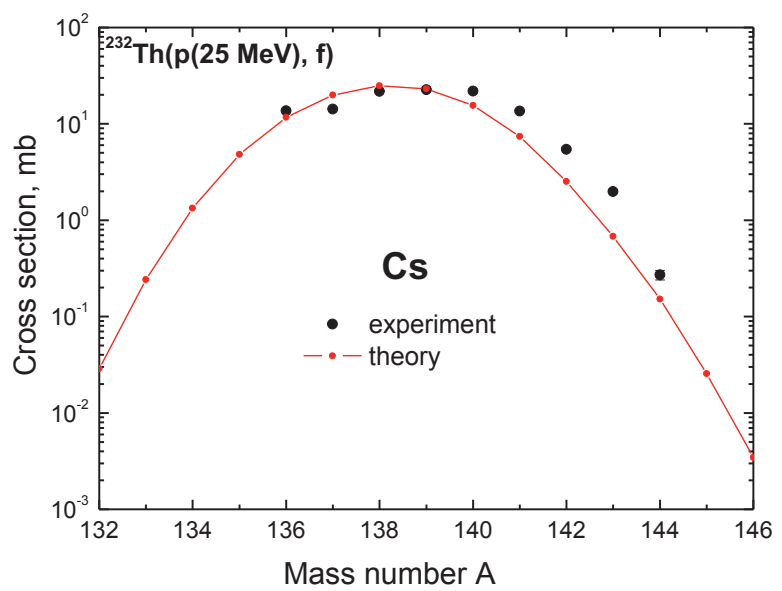

Fig. 9. As in figure 8 for the caesium isotopes. 


\section{$\mathrm{CNR} * 11$}

As seen from figures $6-9$, there is good agreement between calculations and experimental data. The theoretical model provides input data for normalization experimental yields and reproduces such important parameters as FWHM of isotopic distributions and the isotope, which has the highest yield. The high sensitivity of the experimental method allows measuring fission product yields at the level $10^{-2}$ compare to the isotopic maximum, or according to the theoretical model at the level of $10^{-1} \mathrm{mb}$.

\section{Conclusions}

The theoretical model and the experimental method for independent fission products yield measurements are presented in this report. These techniques were used to determine the independent yields in the proton-induced fission of ${ }^{232} \mathrm{Th}$ and ${ }^{238} \mathrm{U}$ at $25 \mathrm{MeV}$. In principle, the same procedure can be applied to study the light particle-induced fission of other targets in the wide energy range of a primary beam.

In experiments almost all nuclides produced in fission with mass numbers $A=76-146$ in the charge interval $\mathrm{Z}=32-56$ were measured up to lower cross section around $0.1 \mathrm{mb}$. The sensitivity presently reached is of the order of $10^{-2}$ as compared to the isotopic maximum. The method is applicable for nuclides with half life longer than the order of $100 \mathrm{~ms}$. For example independent yield of ${ }^{92} \mathrm{Br}$ with $T_{1 / 2}=343 \mathrm{~ms}$ was measured in the experiment with ${ }^{238} \mathrm{U}$ target.

In the present modification, this method is suitable only for relative measurements and it requires additional information to convert results to independent cross-sections. Due to the advantages of this technique, perspectives of the developed method looks quite promising for further developments.

The present model and computer code provide a powerful tool for the experimental data analysis and practically important relevant nuclear data predictions. The calculation method has a special importance for searching experimental method for the extremely neutron-rich nuclide production.

\section{Acknowledgments}

This work is supported by the Academy of Finland under project No. 111428 and No. 139382, the Finnish Center of Excellence Program 2000 - 2005 ( Project No.44875, Nuclear and Condensed Matter Physics Program at JYFL) and the Finnish Center of Excellence Program 2006 - 2011 (Project No. 213503, Nuclear and Accelerator Based Physics Program at JYFL), and by European Union within EURISOL design study.

\section{References}

1. J. Äystö, V. Rubchenya, Eur. Phys. J. A 13, 109 (2002)

2. Final Report of the EURISOL Design Study (2005-2009), Nov. 2009, http://www. eurisol.org/

3. C.D. Bowman et al., Nucl. Instrum. Methods A 320, 336 (1992).

4. J. Bouchard, in Proceedings of the International Conf. on Nuclear Data for Science and Technology, April 22-27, 2007, Nice, France, 2008 CEA, DOI: 10.1051/ndata:07718.

5. H. Penttilä et al. Eur. Phys. J. A 44, 147 (2010).

6. V.A. Rubchenya, Phys. Rev. C 75, 054610 (2007).

7. V.A. Rubchenya et al., Phys. Rev. C 58, 1587 (1998). 
8. V.A. Rubchenya, J. Äystö, Nucl. Phys. A 701, 127c (2002).

9. H. Nifenecker, Journal de Phys.-Lett., France, 41 (1980) L47.

10. V.M. Strutinsky, Nucl. Phys. A 122, 1 (1968).

11. V.V. Pashkevich, Nucl. Phys. A 169, 275 (1971).

12. S. Cwiok, J. Dudek, W. Nazarewicz, J. Skalski, T. Werner, Comp. Phys. Communications 46, 379 (1987).

13. J.P. Bocquet, R. Brissot, Nucl. Phys. A 502, 213c (1989).

14. H.E. Hernandes et al., Nucl. Phys. A 361, 483 (1981).

15. U. Brosa et al., Phys. Rep. 197, 167 (1990).

16. I. Tsekhanovich et al., Phys. Rev. C 70, 044610 (2004).

17. V.A. Rubchenya et al., Nucl. Instrum. Methods Phys. Res., Sect. A 463, 653 (2001).

18. V.A. Rubchenya et al., Nucl. Phys. A 734, 253 (2004).

19. E.M. Kozulin et al., AIP Conf. Proc., vol. 853, p. 336-341, 2006.

20. V.S. Kolhinen et al., Nucl. Instrum. Methods Phys. Res., Sect. A 528, 776 (2004). 